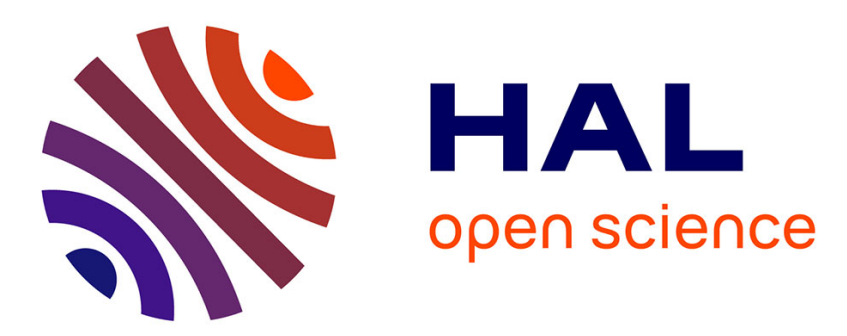

\title{
L'ENTREPRISE, CONSÉQUENCE DE LA SCIENCE MODERNE (1919)
}

Blanche Segrestin

\section{To cite this version:}

Blanche Segrestin. L'ENTREPRISE, CONSÉQUENCE DE LA SCIENCE MODERNE (1919): Présentation d'un extrait de J. Wilbois et P. Vanuxem: Essai sur la conduite des affaires et la direction des hommes. Paris, Payot, 1919. Entreprises et Histoire, 2016, 83 (2), pp.132-136. hal-01336830

\section{HAL Id: hal-01336830 https://hal.science/hal-01336830}

Submitted on 23 Jun 2016

HAL is a multi-disciplinary open access archive for the deposit and dissemination of scientific research documents, whether they are published or not. The documents may come from teaching and research institutions in France or abroad, or from public or private research centers.
L'archive ouverte pluridisciplinaire HAL, est destinée au dépôt et à la diffusion de documents scientifiques de niveau recherche, publiés ou non, émanant des établissements d'enseignement et de recherche français ou étrangers, des laboratoires publics ou privés. 


\title{
L'ENTREPRISE, CONSÉQUENCE DE LA SCIENCE MODERNE (1919)
}

\author{
Présentation de Blanche Segrestin
}

ESKA | «Entreprises et histoire »

2016/2 n 83 | pages 132 à 136

ISSN 1161-2770

ISBN 9782747225984

Article disponible en ligne à l'adresse :

http://www.cairn.info/revue-entreprises-et-histoire-2016-2-page-132.htm

\section{Pour citer cet article :}

Présentation de Blanche Segrestin, « L'entreprise, conséquence de la science moderne (1919) », Entreprises et histoire 2016/2 (n 83), p. 132-136.

Distribution électronique Cairn.info pour ESKA.

(c) ESKA. Tous droits réservés pour tous pays.

La reproduction ou représentation de cet article, notamment par photocopie, n'est autorisée que dans les limites des conditions générales d'utilisation du site ou, le cas échéant, des conditions générales de la licence souscrite par votre établissement. Toute autre reproduction ou représentation, en tout ou partie, sous quelque forme et de quelque manière que ce soit, est interdite sauf accord préalable et écrit de l'éditeur, en dehors des cas prévus par la législation en vigueur en France. Il est précisé que son stockage dans une base de données est également interdit. 


\title{
L'ENTREPRISE, CONSÉQUENCE DE LA SCIENCE MODERNE (1919)
}

\author{
présentation de Blanche SEGRESTIN \\ Centre de Gestion Scientifique \\ École des Mines Paris Tech PSL Research University
}

Le document qui suit est tiré d'un ouvrage de Joseph Wilbois et Paul Vanuxem, publié en 1919, et préfacé par Henri Fayol lui-même :

J. Wilbois et P. Vanuxem, Essai sur la conduite des affaires et la direction des hommes. Paris, Payot, 1919. Bibliothèque politique et économique. Préface de M. H. Fayol.

Joseph Wilbois est un normalien, engagé dans l'éducation des dirigeants. Grand admirateur de Fayol, et qui écrit pour diffuser sa doctrine, il fonde le Club Action et Pensée (CAP) et l'École d'humanités contemporaines en 1913. Puis en 1919 il crée une «École d'administration et d'affaires ». Paul Vanuxem, polytechnicien et membre du corps des ingénieurs des manufactures d'État, pantoufle en 1919 et devient directeur commercial de la société Commentry, Fourchambault et Decazeville (aciéries d'Imphy). Vanuxem est membre de l'Union Sociale des Ingénieurs Catholiques et Wilbois écrit dans son bulletin. Tous les deux sont de la troisième génération leplaysienne. L'ouvrage est publié trois ans après la fondation par Fayol du Centre d'Études
Administratives (CEA) : celui-ci fusionnera ensuite avec la Conférence de l'organisation française pour former le CNOF (Comité national de l'organisation française) et c'est encore Wilbois qu'on y retrouvera comme secrétaire ${ }^{1}$.

L'ouvrage est une forme d'exégèse d'Administration industrielle et générale (1916). Dans leur introduction, Wilbois et Vanuxem prennent soin de mettre en garde les lecteurs de Fayol contre la fausse banalité d'Administration industrielle et générale:

«ce petit volume [Administration industrielle et générale], quand on le parcourt pour la première fois, risque de sembler banal, simplement parce qu'on s'y sent à l'aise, tant sa pensée est voisine de la nôtre ; quand on prend la peine de le méditer, il étonne, illumine, emballe par la richesse des perspectives ouvertes » (p. 12).

Pour démonter cette apparente mais trompeuse simplicité, l'ouvrage part de l'expérience de Fayol et de la «pratique administrative », pour dégager les principes sous-jacents de la théorie administrative. Dans cette optique, et en ligne avec l'idée de

Voir B. Kalaora, « Le mysticisme technique de Joseph Wilbois », in R. Baudouï et Y. Cohen (dir.), Les chantiers de la paix sociale: 1900-1940, Fontenay-aux-Roses, ENS Éditions, 1995 et O. Henry, Les guérisseurs de l'économie. Sociogenèse du métier de consultant (1900-1944), Paris, CNRS Éditions, 2012, p. 177-179, 181, 224-225, 254. 
« tournant fayolien », il insiste sur les ruptures du monde moderne qui sous-tendent la fonction administrative et ses responsabilités.

L'extrait choisi (pages 67-75) porte plus spécifiquement sur l'impact de la science dans le monde des affaires. De manière remarquable, il montre qu'il est indispensable de faire un détour sur les « conditions imposées par la science moderne » pour comprendre, voire pour définir l'entreprise.

«Les conditions imposées par la science moderne "), à savoir la division du travail mais surtout le progrès des méthodes, font que « toute notre production matérielle et morale est tributaire d'une science qui se dépasse sans cesse » (p. 74). L'entreprise découle non seulement de la croissance des organisations, mais aussi de l'intensité du progrès. La science administrative apparaît alors comme la science qui étudie non pas les déterminismes classiques («mouvements d'atomes, d'argent ou d'appétits »), mais ceux qui limitent les inventions quand les hommes s'associent pour des besognes créatrices. Elle porte sur « déterminisme de la liberté ». C'est à cette aune qu'il faut interpréter les principes administratifs fayoliens : le besoin de chef ne croît pas tant avec le nombre de bras qu'avec la vitesse de l'évolution (p. 79). Et avec la science moderne, l'autorité du chef se double de nouvelles responsabilités, notamment celle de développer et de former le personnel.

\section{LA THÉORIE ADMINISTRATIVE - SECONDE PARTIE : LES PRINCIPES}

Avant d'expliquer les principes, nous poserons quelques axiomes de la vie collective, axiomes à la lettre desquels tout le monde adhère, axiomes dont presque personne ne tient compte en esprit, au cours de l'action pratique. Leur oubli est à l'origine de la plupart des fautes d'administration. La méconnaissance des axiomes explique la plupart des maladies administratives dont souffrent nos sociétés d'à présent.

Après les avoir énoncés, nous rappellerons des conditions que la science impose aujourd'hui aux groupements civilisés. Cela nous conduira à la définition de l'entreprise.

C'est aux entreprises ainsi définies que se trouvent appliqués, quand elles réussissent, nos principes.

\section{§I.- LeS AXIOMES de toute VIE} COLLECTIVE

\section{$1^{\circ}$ Axiome du double déterminisme :}

Il y a d'abord dans le monde social deux déterminismes.

Il y a d'abord du déterminisme, sans préciser davantage le sens du mot. Cela tient simplement à ce que la science pénètre nos actes. Sciences physique ou chimique dans les ateliers. Sciences géographique et économique, qui règle l'alimentation en matières premiers et les échanges auxquels les débouchés sont assurés. Science physiologique du travail ouvrier. Psychologie collective des foules en grève. Partout mouvement d'atomes, d'argent ou d'appétits, réglés comme des mouvements d'horloge. Cependant sur ce rythme mécanique chantent des mélodies imprévues. Cela tient aux inventions, techniques pour commencer, 
sociales pour finir. Elles éclatent sans que nul ait pu les prévoir, bousculant, par conséquent, ce déterminisme de première espèce. Mais, en même temps, elles en organisent un second, qui a une tout autre allure. Car ces inventions se réalisent par des groupes d'hommes. Bien que leurs activités ne s'associent que pour des besognes créatrices, elles ne peuvent réussir à se surpasser elles-mêmes qu'en se soumettant à certains usages qui, pour n'avoir pas l'aspect fatal ou numérique des lois de la matière, n'en constituent pas moins des limitations à la fantaisie : limitations qu'il faut connaître et respecter si l'on veut que ces activités se développent en un faisceau efficace au lieu d'avorter dans une interférence de caprices. C'est du déterminisme, non parce qu'on est déterminé, mais parce qu'on se détermine en se mettant dans les conditions où la spontanéité est le plus à l'aise pour s'épanouir. C'est ce qu'on a appelé, dans un ouvrage où on l'étudiait de plus près, le déterminisme de la liberté .

\section{$2^{\circ}$ Cinq axiomes de la vie collective $e^{3}$ :}

Premier axiome. La vie collective n'est pas le fait d'un seul, mais celui d'une collection de volontés. - Une machine est la chose du mécanicien. Les rouages inertes ne peuvent que transformer, en la dégradant, une énergie venue d'ailleurs. Mais une société n'a de vie que dans la mesure où ses membres consentent à dépenser à son service une énergie à eux personnelle, et donc chacun tient en lui-même à la fois le contrôle et la source. Cela était vrai dans l'antique esclavage : que pouvaient les châtiments sur l'esclave résiné à se laisser tuer sur place? C'est vrai bien davantage aujourd'hui : la part de décision du chef a diminué au profit d e a part de décision de la troupe ; l'inférieur obéit moins qu'il ne collabore.
Décider chaque individu à l'effort pour qu'il exalte son rendement personnel, voici une première tâche pour la fonction administration qui « stimule », " rémunère », encourage l'initiative », répand le « goût des responsabilités $\gg \ldots$

Deuxième axiome. La vie collective suppose l'harmonie e et l'harmonie sociale ne se crée pas toute seule. - Le rendement social n'est pas une somme arithmétique de rendements individuels : une collection de bonnes volontés non "polarisées », c'est l'anarchie, c'est l'impuissance du néant. Mais l'harmonie que l'homme trouve toute faite dans les êtres de la nature, il faut qu'il la crée dans les sociétés qu'il forme comme il la met dans les machines. La cohésion sociale n'est pas un fait spontané, et bien que beaucoup d'hommes vivent sans aucun souci de l'ordre, comme si l'ordre se faisait tout seul, l'ordre n'existe que dans la mesure où la fonction administrative y pourvoit.

Faire converger les efforts individuels, telle est la seconde tâche de la fonction administrative, de sens opposé à la première, mais non moins indispensable à la vie sociale.

Troisième axiome. Pour créer la vie sociale, la fonction administrative a pour point d'appui la connaissance positive. - on ne commande aux choses et aux hommes qu'à la condition de les bien connaître. On ne manœuvre les choses qu'en respectant leurs habitudes parce qu'il y a en nous de mécanique, nous sommes des choses. Il faut à l'administrateur le sentiment profond du déterminisme, la foi dans la connaissance scientifique. Le sens des réalités, voilà la forme première du bon sens, pour qui prétend administrer et c'est peut être la moins répandue parmi les administrateurs en place. Les ordres inexécutables, les règlements impossibles à observer, les lois inopérantes

J. Wilbois, Devoir et Durée. Essai de morale sociale, Paris, Alcan, 1912, Chap. VI et VIII.

Cf. P. Vanuxem, Industrialiser. Introduction théorique et pratique à l'étude de l'administration expérimentale, Paris, Dunod et Pinat, 1918. Les hypothèses implicites d'Administration industrielle et générale. 
sont le fruit ordinaire de l'administration $a$ priori : les faits dont les créateurs de systèmes négligent la préliminaire inspection ont pour coutume de se venger comme naguère les fées oubliées au baptême.

Prendre et garder le contact intellectuel avec les choses et les hommes, connaître avec méthode, administrer commence par là.

Quatrième axiome. Pour créer la vie sociale, la fonction administrative s'exerce par des procédés exprès. Pas d'administration sans procédés. - Comme toute autre action, l'action administrative n'est efficace que si elle est méthodique. On ne prévoit pas sans "programme », on ne coordonne pas sans « conférence », ou quelque "procédé » qui en tienne lieu. Il faut à l'administrateur des outils pour brasser la masse sociale, pour faire parvenir l'action de la volonté « polarisatrice » jusqu'aux limites de la masse à « polariser ».

Prendre et garder le contact pratique avec les choses et les hommes, agir avec méthode, administrer est là, tout entier.

Cinquième axiome. L'opération administrative doit être universelle et permanente. - Aucune part, aucun endroit, aucun moment de la vie sociale ne peut être soustrait sans dommage à la fonction administrative, qui est « création » et " providence » tout à la fois. Pour le corps social, s'administrer, c'est vivre tout simplement.

Ces axiomes sont de tous les temps, de tous les pays, et ils s'appliquent à toutes les formations sociales. Mais l'Occident contemporain connaît, en outre, certaines conditions qui lui viennent de sa technique et qui, conjuguées aux axiomes, donneront les « principes » que nous trouverons appliqués à nos entreprises.

§2.- CONDITIONS IMPOSÉES PAR LA SCIENCE MODERNE
Première condition donnée. La division et la coordination du travail. - La division du travail prend plusieurs formes. Elle est d'abord géographique : le blé, la betterave, la vigne, se cultivent de plus en plus dans les régions qui donnent le meilleur rendement. Elle est ensuite technique : pour faire une paire de chaussures, il y a des dizaines d'opérations différentes, confiées chacune à des ouvriers spécialistes. Elle est aussi scientifique : un chimiste ne connaît qu'un chapitre de la chimie, ou plutôt qu'un paragraphe. Elle est encore politique, le père de famille ayant perdu la plupart de ses antiques fonctions de prêtre, de juge ou de chef d'atelier. Il faut ajouter que dès que des fonctions qui se trouvaient autrefois fondues en un même individu, se répartissent en des hommes différents, il se crée ou il faut qu'on crée entre eux des liens d'autant plus solides que la spécialisation est plus avancée ; division du travail postule coordination du travail; ici le mot diviser doit être traduit par réunir.

Deuxième condition donnée. Le progrès des méthodes. - c'est avant tout le machinisme qui a décuplé, parfois centuplé la production, et prodigieusement réduit les distances, par les chemins de fer et les paquebots. Mais c'est aussi l'ensemble de tous les procédés actuels de l'industrie, engrais artificiels, électrométallurgie, synthèses chimiques. Même perfectionnement dans les méthodes de la pure spéculation, analyse d'une étoile par le spectroscope, reconstitution d'un psaume hébreu en tenant compte des règles de la poésie hébraïque. Même perfectionnement dans la vie politique, dès qu'on commence à faire précéder les réformes législatives d'enquêtes où l'on se sert de données systématisées, soit monographiques, soit statistiques. On peut dire que toute notre production matérielle et morale est tributaire d'une science qui se dépasse sans cesse. 
Première conséquence: l'échelle des entreprises augmente, et cela dans un double sens.

a) Chaque entreprise, en un lieu donné, croît. Cela tient à ce que l'on sait fabriquer des machines nécessitant de plus nombreux servants, à ce que les grands établissements, où l'on peut récupérer des déchets infimes et qui supportent les frais de laboratoires d'essais, coûtent moins cher, et aussi à ce que la spécialisation de la terre par régions, reliées grâce à des moyens de transports supérieurs, suscite en chaque point des établissements semblables, qui se font une concurrence où les petits crouleraient, à moins d'une entente. Les exemples sont connus: ce sont les aciéries Carnegie ou les filatures Morosoff, qui sont des villes, et des villes importantes; c'est la demi-douzaine de grands magasins qui suffisent à alimenter Paris ; une légion d'informateurs aident des géographes qui ont entrepris l'étude de tous les pays de la France; un chef d'armée commande à des millions de soldats. L'anarchie sociale ne peut être qu'une survivance. Nous sommes au siècle de la concentration.

b) Mais, en même temps, chaque entreprise a des attaches sur toute la surface du globe. Cela tient à ce que la division du travail géographique place à d'énormes distances les différentes sections d'une même affaire. Une société métallurgique peut avoir ses mines de houille fort loin de ses mines de fer. La météorologie a ses laboratoires partout. Les sociétés antituberculeuses de tous les pays unissent leurs informations. Nous entrons - pour employer un barbarisme expressif - dans l'âge du mondialisme.

La seconde conséquence, c'est la rapidité de l'évolution. - Elle tient à l'acuité de nos instruments de découvertes, et cette acuité est encore exaspérée par la fièvre des relations internationales. Les objets qui nous servent le plus sont des inventions des toutes dernières années, l'aéroplane, le ciment armé, les sérums. Ces brusques inventions ont produit des révolutions économiques dans des pays entiers : l'Inde cesse d'être tributaire de l'Angleterre pour ses tissus et même pour ses métiers. Les révolutions économiques déclenchent des révolutions sociales : la caste se dissout devant la hiérarchie de l'usine ou la cohésion du syndicat. Partout, c'est une trépidation qui déconcerte les vieilles autorités. L'expérience d'une génération ne profite guère à la suivante. Le dieu du temps, pour traverser ce siècle, est monté sur une cent chevaux.

Le vingtième siècle, par ses transformations, nous a singulièrement armés : il a donné leur plein épanouissement à des groupes caractéristiques de notre temps et que nous nommons entreprises. 